\title{
Multiscale Analysis of Solar loading Thermographic Data for the Inspection of Civil Engineering Structures
}

\author{
by Katherine $\mathrm{Tu}^{\star}$, Stefano Sfarra**, Clemente Ibarra-Castanedo ${ }^{\star \star \star}$, Xavier P.V. Maldague ${ }^{\star \star \star}$, Yuan Yao** \\ * Department of Chemical Engineering, National Tsing Hua University, Hsinchu, 30013, Taiwan, ROC, \\ ** Department of Industrial and Information Engineering and Economics, University of L'Aquila, Piazzale E. \\ Pontieri n. 1, Monteluco di Roio - L'Aquila (AQ), I-67100, Italy \\ ***Computer Vision and Systems Laboratory, Laval University, Quebec City, Quebec, G1K 7P4, Canada \\ Corresponding author: yyao@mx.nthu.edu.tw
}

\begin{abstract}
The concept of multiscale thermographic data processing is proposed in this work for the inspection of civil engineering structures. In the proposed framework, thermograms of buildings collected by solar loading thermography are decomposed into a number of intrinsic mode functions under different scales by multidimensional ensemble empirical mode decomposition (MEEMD). Then, principal component analysis (PCA) is adopted to extract the features at each scale. Compared to the popular principal component thermography (PCT) method that applies PCA to raw thermographic data, multiscale analysis provides an opportunity to zoom in on different types of structural features.
\end{abstract}

\section{Introduction}

Infrared thermography is a widely adopted non-destructive testing method, where the thermograms obtained by infrared cameras capture the surface temperature distributions of the investigated targets through time so the information of both the surface and internal structures can potentially be extracted. Although some obvious structural information can be obtained by the naked-eye observation of the raw (unprocessed) thermograms, data processing is usually a necessity in order to improve the detection results [1-6]. Among the popular techniques, principal component thermography (PCT) [7] and its extensions [8-11] have been widely implemented in different fields because of their good performance in noise reduction, data compression, and feature extraction.

Recently, solar loading thermography was demonstrated to be a promising method for the inspection of civil engineering structures [12], where the surface temperature evolution of the inspected object subjected to solar irradiation and environmental temperature changes are recorded with an infrared camera. After data acquisition, advanced signal processing methods, such as PCT [7], greatly improve the contrast of the structural features of the investigated objects. Nevertheless, the existing thermographic data processing methods seldom consider the multiple spatial scale issue that is an inherent characteristic of civil engineering structures. As a result, some structural details may not be revealed.

In the past, multi-dimensional ensemble empirical mode decomposition (MEEMD) [13] was successfully applied to the inspection of polymer composite materials [14] and mosaics [15]. In those works, MEEMD was implemented to each thermogram for signal decomposition. In doing this, the high-frequency noise, the low-frequency backgrounds, and the feature-related information are separated into a number of intrinsic mode functions (IMFs). Consequently, the material features can be visually identified by reconstructing these IMFs into a number of component images. However, further analysis of the decomposition result of every thermogram captured in infrared testing is a time-consuming and laborious task.

In this work, a multiscale thermographic data processing framework is proposed, which uses MEEMD to decompose each thermogram collected in the testing experiments into a series of IMFs corresponding to different spatial scales and then adopts PCA [16] to extract the information contained in each scale. Experimental results on the processing of solar loading thermographic data show that the proposed method can be considered as an interesting complement to the conventional PCT.

\section{Methodologies}

\subsection{Solar loading thermography}

Solar loading thermography is a combination of both passive and active infrared testing approaches, where the main energy source is solar irradiation while environmental temperature changes also affect the surface temperature evolution of the investigated object. Such an energy source cannot be precisely controlled, because of the environmental weather effects. As in other types of infrared testing, the surface temperature of the inspected object is recorded by an infrared camera during the testing time period. Then, the acquired thermographic data, i.e. the thermograms, are usually processed with signal processing, statistical, or machine learning methods for noise reduction, contrast improvement, and feature extraction, which is useful for characterization of the surface/subsurface features or defects. 


\subsection{Decomposition with multi-dimensional ensemble empirical mode decomposition}

When applied to civil engineering applications, the solar loading thermography testing often result in thermograms containing multi-spatial scale information, because multiple spatial scales are an inherent characteristics of most civil engineering structures. Here, we propose to integrate MEEMD and PCA to achieve multiscale analysis of the solar loading thermographic data. The flowchart is shown in Fig. 1.

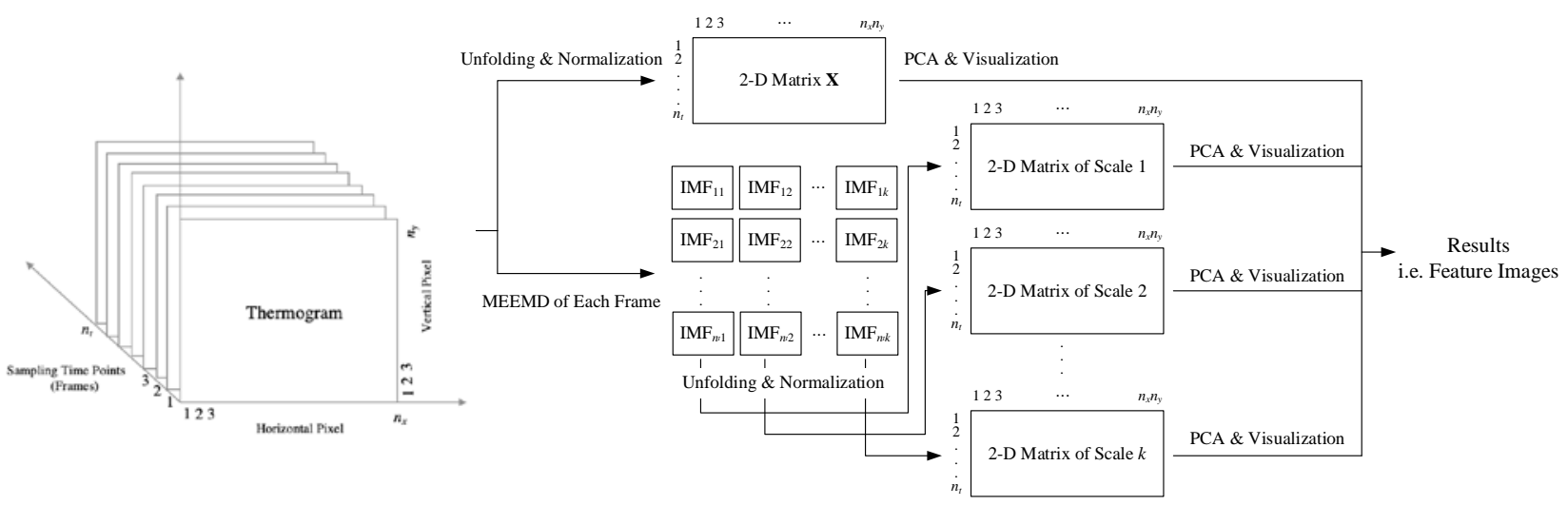

Fig. 1. Flowchart of Multiscale Thermographic Data Analysis

After the thermograms are collected, MEEMD is implemented to decompose each thermogram into different spatial scales. The fundamental of MEEMD is the empirical mode decomposition (EMD) method [17] that is a nonparametric adaptive data analysis method. Compared with the wavelet-based mode decomposition methods [18], EMD does not require user-specified parameters and can be adopted to handle nonlinear and nonstationary problems. For a one-dimensional (1-D) signal $x$, its EMD results can be represented by the following equation:

$$
x=\sum_{j=1}^{J} c_{j}+r
$$

where $c_{j}$ is the jth IMF decomposed from the original signal $x$ sorting from the highest frequency to the lowest frequency, $r$ is the residue of the original data. The IMFs provide information in different scales, which are functions satisfying the following requirements [19].

(a) The number of extrema and the number of zero-crossings must either be equal or differ at most by one.

(b) At any point, the mean value of the upper and lower envelopes defined by the local maxima and local minima is zero.

The procedure of extracting an IMF is called sifting. For a 1-D signal $x(t)$, the sifting process of the conventional EMD can be briefly described as following.

(1) Let the data $S(t)=x(t)$.

(2) Identify all the local extrema in $S(t)$.

(3) Connect all the local maxima by a cubic spline line to generate the upper envelope.

(4) Connect all the local minima by a cubic spline line to generate the lower envelope.

(5) Calculate the mean of the two envelops which is denoted as $m(t)$. This produces a lower frequency component than the original signal.

(6) Subtract $m(t)$ from $S(t)$ to obtain an oscillatory signal $h(t)$.

(7) Check if $h(t)$ satisfies the requirements for an IMF.

(8) If the conditions of IMF are not satisfied, $h(t)$ is treated as the data, i.e. $S(t)=h(t)$, and the above steps are repeated.

(9) Otherwise, an IMF is obtained, i.e. $c_{1}(t)=h(t)$.

(10) Treat the residue between the original data $x(t)$ and all obtained IMFs as the new data and repeat the previous steps to find out all the IMFs.

(11) This iterative procedure is terminated when the residue $r$ becomes a monotonic function.

A major limitation of EMD is due to the mode mixing problem [20], which means that a single IMF consists of multiple different levels of signal interferences or that the same level of signals occurs in different IMFs. The cause of this problem is the intermittency phenomenon in the signal. In such cases, ensemble empirical mode decomposition (EEMD) [20] method is a useful alternative.

EEMD is a type of noise-assisted data analysis method, which repeats EMD multiple times and achieves a "true" IMF by averaging the corresponding IMFs obtained in different trials. In each trial, EMD is conducted on a generated signal that is a sum of the original signal and a series of white noise with a finite amplitude. By taking average, the mode mixing 
effects and the noises are cancelled out and the decomposition results are more reasonable.

The EEMD method was further extended to a multi-dimensional version, i.e. MEEMD [13]. Herein, it is used to deal with two-dimensional (2-D) signals, i.e. the thermograms recorded in the solar loading thermography experiments. As shown in Fig. 1, each thermogram can be regarded as a 2-D signal whose two dimensions are horizontal pixels (x-direction) and vertical pixels (y-direction), respectively. In another point of view, each thermogram is a collection of a series of 1-D signals in $\mathrm{x}$ - or $\mathrm{y}$-direction. In MEEMD, EEMD is applied to each $\mathrm{x}$-direction signal and decomposes it to $J_{x}$ IMFs. As a result, a thermogram is decomposed to $k$ images. Then, EEMD is implemented again to decompose each y-direction signal in these images into $J_{y}$ IMFs. These IMFs are reconstructed to $J_{x} J_{y}$ sub-images each of which is denoted as $h_{j, i}$, where $j=1, \ldots, J_{x}$ and $i=1, \ldots, J_{y}$. Then, the sub-images are recombined into $k=\min \left(J_{x}, J_{y}\right)$ component images, where the $i$ th component can be calculated as

$$
C_{i}=\sum_{j=i}^{J_{y}} h_{i, j}+\sum_{j=i+1}^{J_{x}} h_{j, i}
$$

In doing this, each thermogram is decomposed into $k$ component images with a descending order of spatial frequency, providing a multiscale view of the thermographic data. For more details of the MEEMD algorithm, please refer to the cited paper [13].

\subsection{Processing with principal component analysis}

Supposing that there are $n_{t}$ thermograms collected in the experiments (typically several hundreds), $n_{t} k$ component images are obtained after conducting MEEMD. Investigating each of these component images with naked eyes is laborious and impractical. Therefore, it is desired to extract the information from the images corresponding to each scale by dimensionality reduction. Here, PCA is used for further analysis.

In previous research, PCA was implemented to process thermographic data and summarize defect information contained in the raw thermograms. Such a method is named PCT [7]. In the first step of PCT, the entire thermographic dataset is transformed to a 2-D data matrix by vectorising each thermogram. After data normalization, PCA is implemented, whose mathematical expression is as follows.

$$
X=T P^{T}
$$

where $X$ is one of the normalized 2-D data matrix obtained in the previous steps and $T$ is the score matrix each column of which is a vector of principal component (PC). The first PC explains the largest fraction of the variance in the dataset. The second PC is orthogonal to the first one and contains most of variation information that is not explained by the first PC, and so on. Therefore, most systematic variation information is expressed by the first several PCs. $P$ is the loading matrix describing the transformation relationship between $X$ and $T$, which reflects the correlation between the pixels in the thermograms. Then, the loading vectors corresponding to the first several PCs are reshaped and visualized as a series of feature images. In doing this, it is not necessary to exam all the raw thermograms. Instead, only a small number of feature images should be checked.

Herein, we apply PCA in a similar way to PCT to accomplish multiscale thermographic data analysis. Denote the $j$ th MEEMD component decomposed from the ith thermogram as $c_{j}^{i}$. Construct a 2-D matrix $X_{j}$ in which the ith row is the vectorised $c_{j}^{i}$. Supposing that $X_{j}$ has been normalized, PCA can be applied to this matrix and the loading matrix $P_{j}$ is calculated. By reshaping the first several columns in each $P_{j}$ matrix $(j=1, \ldots, k)$ to matrices with size of $n_{x} \times n_{y}$ and visualizing each of them, the important features in different scales are highlighted. It is noted that the conventional PCT can also be implemented. The multiscale analysis results are the supplement of conventional PCT.

\section{Experimental Results}

\subsection{Raw thermograms}

In the experiment of solar loading thermography, a series of thermograms were obtained to inspect the civil engineering structures of a building at Laval University campus (Canada). Fig. 2 shows a photo of the inspected building area. In addition to the surface structures, there are two internal structure signatures that are of interest, including a sealed door and the floor's slab as marked in the thermogram shown in Fig. 3(a). The area of the region of interest is $128 \times 128$ pixels. The blue coloured region at the right bottom is a tree in the foreground. All four subplots in Fig. 3 are the raw thermograms recorded by a long-wave infrared microbolometer at different time points. It is clear that most of the thermograms do not show distinguishable boundary of the slab. In the meantime, the surface characteristics at the small spatial scale, such as the ceramic tiles, are almost invisible in the raw thermograms. Therefore, the advanced thermographic data decomposition and analysis methods are necessary to improve the identification of the detailed civil engineering structures. 


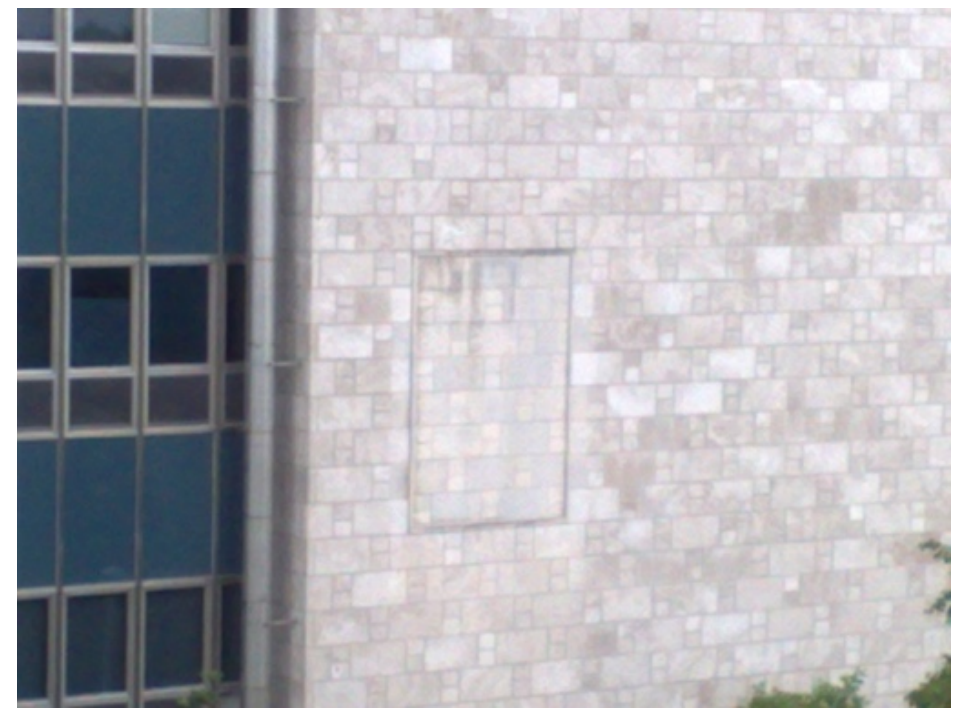

Fig. 2. View of inspected wall.

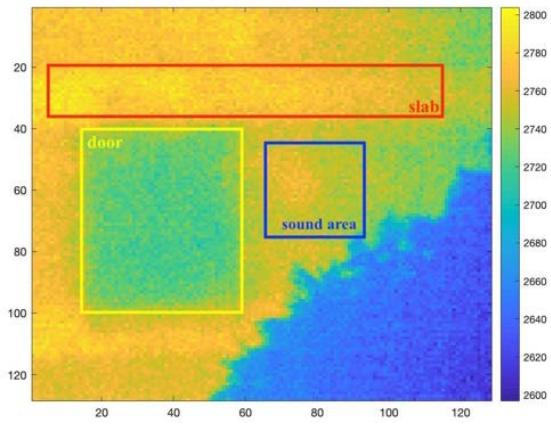

(a)

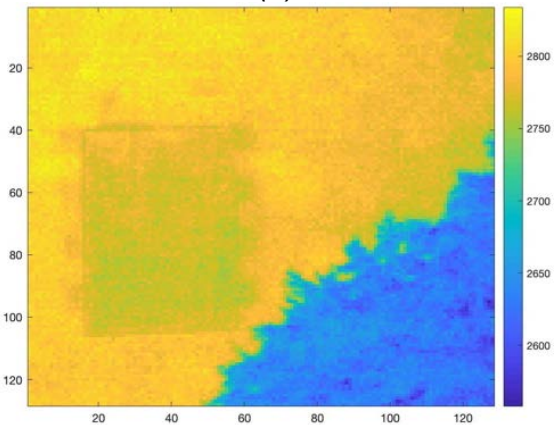

(c)

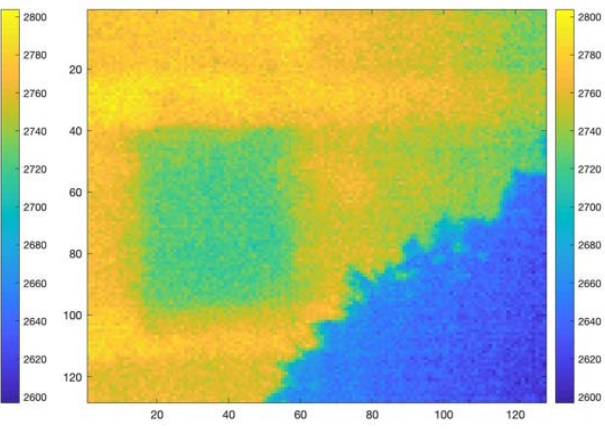

(b)

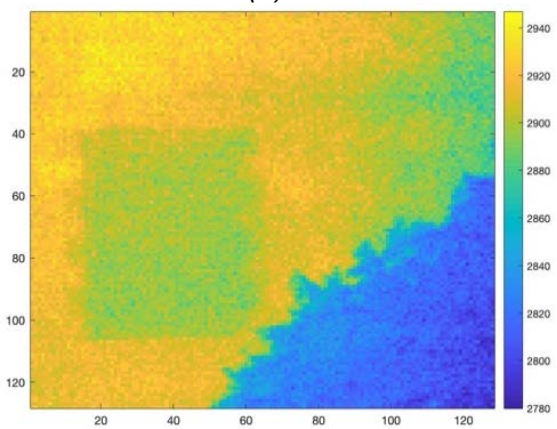

(d)

Fig. 3. Raw thermograms acquired at different time points

\subsection{Decomposition of thermograms}

MEEMD was implemented to process 38 thermograms. Each thermogram was decomposed to five components each of which reflects the features at a different scale, i.e. a different spatial frequency. Fig. 4 shows the decomposition results of two thermograms, where the component images corresponding to high frequencies are plotted on the top while the signals with progressively lower frequencies are visualized towards the bottom. It is observed that the component image at the highest frequency is very noisy, while the lowest frequency highlights the foreground of the thermograms. Some structure information is extracted by the component images at the middle frequency. However, it is hard to distinguish between the interested structures and the surroundings. In addition, it is difficult to explore in detail all the component images because of the large amount of them. 


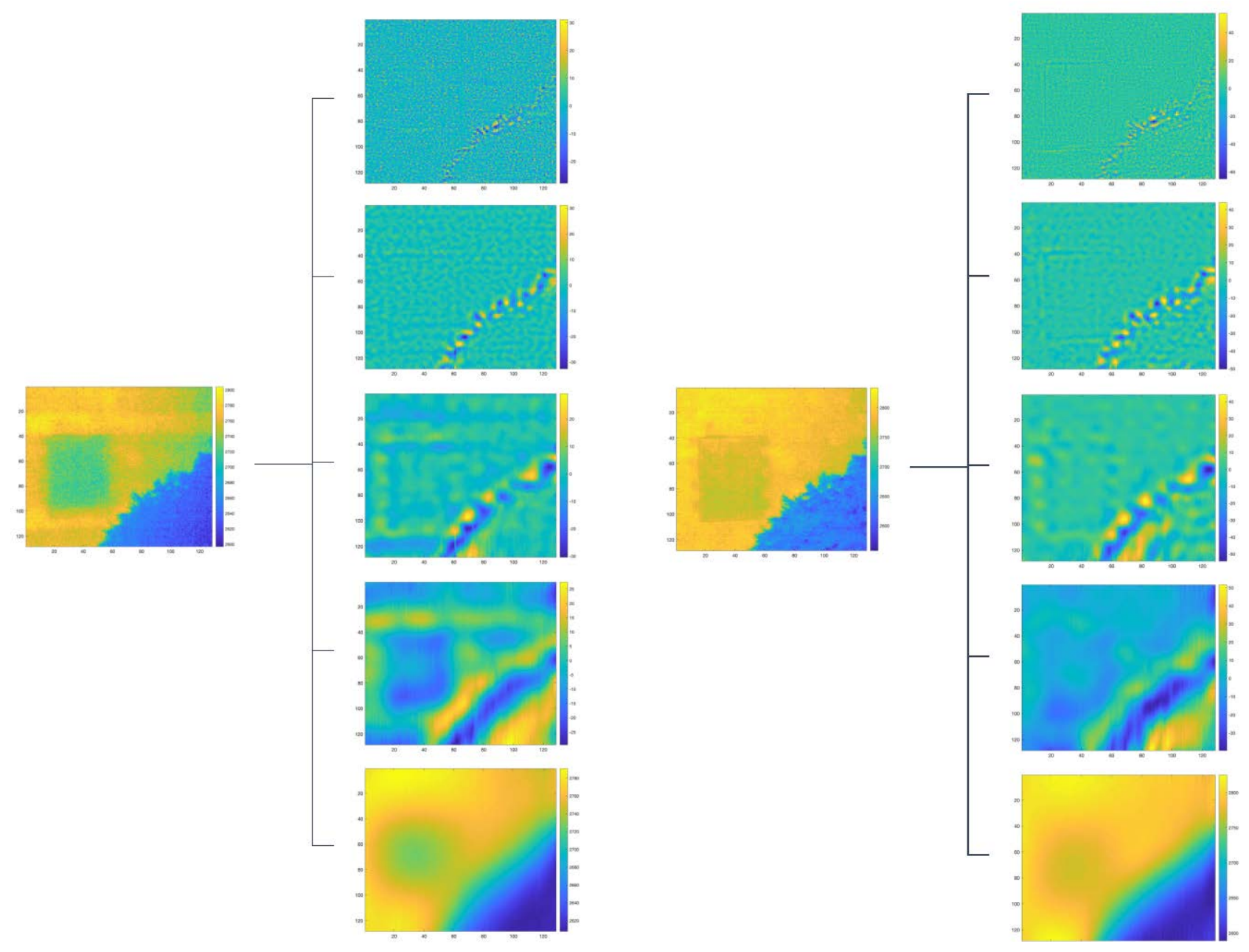

Fig. 4. MEEMD results

\subsection{Multiscale data processing}

In the next step, PCA was used to further extract features from both the raw thermograms and the MEEMD component images at different scales. Fig. $\mathbf{5}$ shows three loading images of PCT. Obviously, compared to the raw thermograms, the PCT results present an improved contrast between the slab and the surrounding. In addition, the sealed door can be viewed clearly.
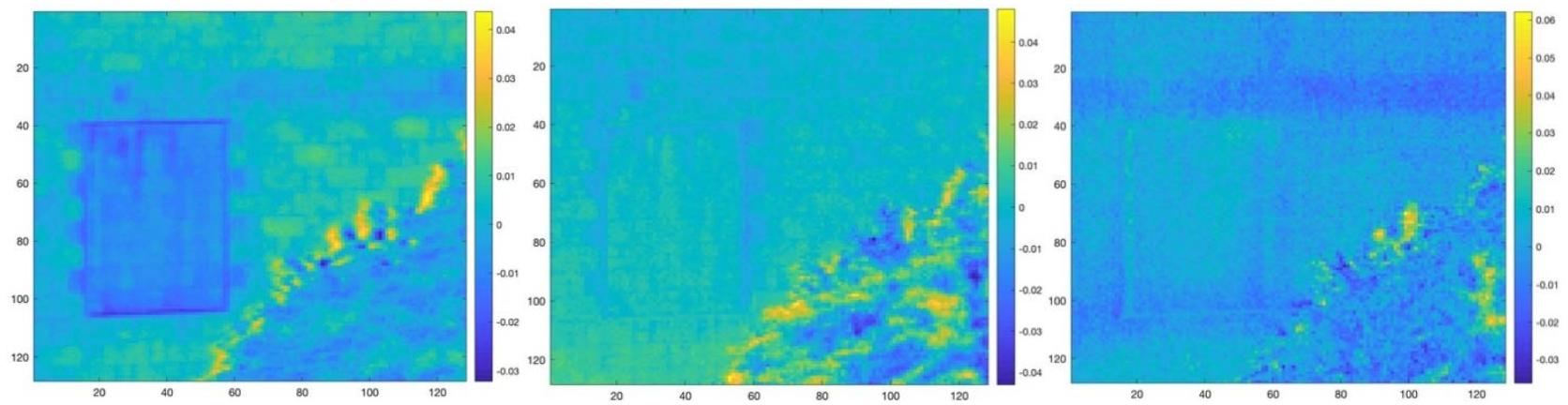

Fig.5. PCA loading images of raw thermograms, i.e. PCT results

More detailed structures of the surface features are clearly displayed in the multiscale analysis results. The first two results in Fig. 6 are the loading images obtained by applying PCA on the IMF signals corresponding to the highest frequencies, which show the outlines of the ceramic tiles clearly. The rightmost image shows the results corresponding to the lowest spatial frequency, which distinguishes the foreground. 

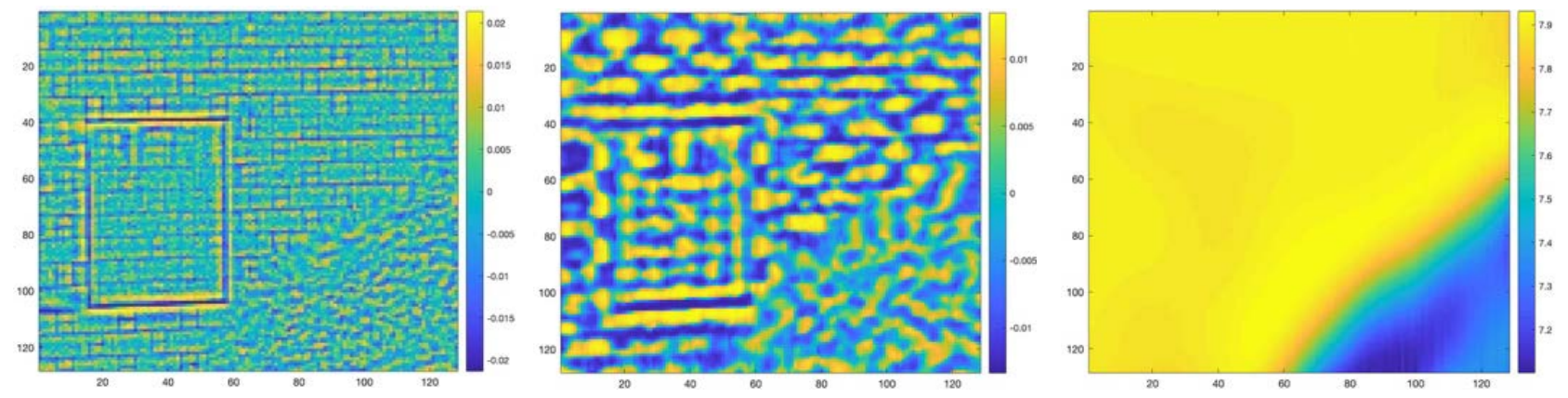

Fig. 6: PCA Loading Images of IMF Signals Corresponding to Highest Frequencies and Lowest Frequency

\section{Conclusions}

In this paper, a multiscale thermographic data analysis method is proposed for the inspection of the civil engineering structures. By integrating the benefits of MEEMD and PCA, the proposed method provides an opportunity to zoom in on different types of structural features, whose feasibility is illustrated with the experimental results.

\section{Acknowledgments}

This work was supported in part by Ministry of Science and Technology, ROC, under Grant MOST 108-2221-E007-068-MY3.

\section{REFERENCES}

[1] Maldague X., Galmiche F., Ziadi A. Advances in pulsed phase thermography. Infrared Physics \& Technology, 43: 175-181, 2002.

[2] Shepard S. M. Reconstruction and enhancement of active thermographic image sequences. Optical Engineering, 42: 1337-1342, 2003.

[3] Madruga F. J., Ibarra-Castanedo C., Conde O. M., López-Higuera J. M., Maldague X. Infrared thermography processing based on higher-order statistics. NDT \& E International, 43: 661-666, 2010.

[4] Zheng K., Chang Y.-S., Yao Y. Defect detection in CFRP structures using pulsed thermographic data enhanced by penalized least squares methods. Composites Part B: Engineering, 79: 351-358, 2015.

[5] Zheng K., Chang Y.-S., Wang K.-H., Yao Y. Improved non-destructive testing of carbon fiber reinforced polymer (CFRP) composites using pulsed thermograph. Polymer Testing, 46: 26-32, 2015.

[6] Yan Z., Chen C.-Y., Luo L., Yao Y. Stable principal component pursuit-based thermographic data analysis for defect detection in polymer composites. Journal of Process Control, 49: 36-44, 2017.

[7] Rajic N. Principal component thermography for flaw contrast enhancement and flaw depth characterisation in composite structures. Composite Structures, 58: 521-528, 2002.

[8] Yousefi B., Sfarra S., Ibarra Castanedo C., Maldague X. Comparative analysis on thermal non-destructive testing imagery applying Candid Covariance-Free Incremental Principal Component Thermography (CCIPCT). Infrared Physics \& Technology, 85: 163-169, 2017.

[9] Wu J., Sfarra S., Yao Y. Sparse principal component thermography for subsurface defect detection in composite products. IEEE Transactions on Industrial Informatics, 14: 5594-5600, 2018.

[10] Yousefi B., Sfarra S., Sarasini F., Castanedo C. I., Maldague X. Low-rank sparse principal component thermography (sparse-PCT): Comparative assessment on detection of subsurface defects. Infrared Physics \& Technology, 98: 278-284, 2019.

[11] Jie J., Dai S., Hou B., Zhang M., Zhou L. Defect Detection in composite products based on sparse moving window principal component thermography. Advances in Polymer Technology, 2020: Article ID 4682689, 2020.

[12] Ibarra-Castanedo C., Sfarra S., Klein M., Maldague X. Solar loading thermography: Time-lapsed thermographic survey and advanced thermographic signal processing for the inspection of civil engineering and cultural heritage structures. Infrared Physics \& Technology, 82: 56-74, 2017.

[13] Wu Z., Huang N. E., Chen X. The multi-dimensional ensemble empirical mode decomposition method. Advances in Adaptive Data Analysis, 01: 339-372, 2009.

[14] Chang Y.-S., Yan Z., Wang K.-H., Yao Y. Non-destructive testing of CFRP using pulsed thermography and multi-dimensional ensemble empirical mode decomposition. Journal of the Taiwan Institute of Chemical Engineers, 61: 54-63, 2016.

[15] Yao Y., Sfarra S., Ibarra-Castanedo C., You R., Maldague X. The multi-dimensional ensemble empirical mode decomposition (MEEMD): An advanced tool for thermographic diagnosis of mosaics. Journal of Thermal Analysis and Calorimetry, 128: 1841-1858, 2017. 
[16] Wold S., Esbensen K., Geladi P. Principal component analysis. Chemometrics and Intelligent Laboratory Systems, 2: 37-52, 1987.

[17] Huang N. E., Shen Z., Long S. R., Wu M. C., Shih H. H., Zheng Q., Yen N.-C., Tung C. C., Liu H. H. The empirical mode decomposition and the Hilbert spectrum for nonlinear and non-stationary time series analysis. Proceedings of the Royal Society of London. Series A: Mathematical, Physical and Engineering Sciences, 454: 903-995, 1998.

[18] Torrence C., Compo G. P. A practical guide to wavelet analysis. Bulletin of the American Meteorological Society, 79: 61-78, 1998.

[19] Hu X., Peng S., Hwang W. EMD revisited: a new understanding of the envelope and resolving the modemixing problem in AM-FM signals. IEEE Transactions on Signal Processing, 60: 1075-1086, 2012.

[20] Wu Z., Huang N. E. Ensemble empirical mode decomposition: a noise-assisted data analysis method. Advances in Adaptive Data Analysis, 1: 1-41, 2009. 